\title{
Generalized Contraction Principle in Complex valued Metric spaces
}

\author{
Parveen Kumar ${ }^{1}$, Sanjay Kumar ${ }^{2}$ \\ ${ }^{1,2}$ Department of Mathematics, Deenbandhu ChhotuRam University of Science and Technology, Murthal, \\ Sonepat-131039, Haryana (India)
}

Abstract:In this paper, we introduce the notion of Generalized contractive type mappings in complex valued metric space and establish fixed point theorem for these mappings.

Keywords: Complex valued metric space, Generalized contractive maps,fixed point.

\section{Introduction}

The existence and uniqueness of fixed point theorems of operators or mappings has been a subject of great interest since the work of Banach in 1992[2].The Banach contraction mapping principle is widely recognized as the source of metric fixed-point theory. A mapping $\mathrm{T}: \mathrm{X} \rightarrow \mathrm{X}$, where $(\mathrm{X}, \mathrm{d})$ is metric space, is said to be contraction mapping if for all $\mathrm{x}, \mathrm{y} \in \quad \mathrm{X}, \quad \mathrm{d}(\mathrm{Tx}, \mathrm{Ty}) \leq \lambda \mathrm{d}(\mathrm{x}, \mathrm{y}), \quad 0<\lambda<1$. According to the Banach contraction mapping principle, any mapping T satisfying (1) in Complete metric space will have a unique fixed-point. This principle includes different directions in different spaces adopted by mathematicians; for example ,metric space, G-metric spaces, partial metric spaces,cone metric spaces have already been obtained. A new space called the complex valued metric space which is more general than wellknown metric space has been introduced by Azam et.al. Azam proved some fixed-point theorems for mappings satisfying a rational inequality. In2012, Rouzkard and Imdad [3] extended and improved the common fixedpoint theorems which are more general than the result of Azam et.al. [1]. The idea of complex valued metric spaces can be exploited to define complex valued normed spaces and complex valued Hilbert spaces; additionally,it offers numerous research activities in mathematical analysis.

\section{Basic Facts and Definitions}

We recall some notations and definitions which will be utilized in our discussion.

Let $\mathbf{C}$ be a set of complex numbers and $\mathrm{z}_{1}, \mathrm{z}_{2} \in \mathbf{C}$. Define a partial order $\leqslant$ on $\mathbf{C}$ as follows:

$\mathrm{z}_{1} \leqslant \mathrm{z}_{2}$ if and only if $\operatorname{Re}\left(\mathrm{z}_{1}\right) \leq \operatorname{Re}\left(\mathrm{z}_{2}\right), \operatorname{Im}\left(\mathrm{z}_{1}\right) \leq \operatorname{Im}\left(\mathrm{z}_{2}\right)$.

It follows that $z_{1} \leqslant z_{2}$ if one of the following conditions is satisfied:

(i) $\operatorname{Re}\left(\mathrm{z}_{1}\right)=\operatorname{Re}\left(\mathrm{z}_{2}\right), \operatorname{Im}\left(\mathrm{z}_{1}\right)<\operatorname{Im}\left(\mathrm{z}_{2}\right)$.

(ii) $\operatorname{Re}\left(\mathrm{z}_{1}\right)<\operatorname{Re}\left(\mathrm{z}_{2}\right), \operatorname{Im}\left(\mathrm{z}_{1}\right)=\operatorname{Im}\left(\mathrm{z}_{2}\right)$.

(iii) $\operatorname{Re}\left(\mathrm{z}_{1}\right)<\operatorname{Re}\left(\mathrm{z}_{2}\right), \operatorname{Im}\left(\mathrm{z}_{1}\right)<\operatorname{Im}\left(\mathrm{z}_{2}\right)$.

(iv) $\operatorname{Re}\left(\mathrm{z}_{1}\right)=\operatorname{Re}\left(\mathrm{z}_{2}\right), \operatorname{Im}\left(\mathrm{z}_{1}\right)=\operatorname{Im}\left(\mathrm{z}_{2}\right)$.

In (i), (ii) and(iii), we have $\left|z_{1}\right|<\left|z_{2}\right|$.In(iv), we have $\left|z_{1}\right|=\left|z_{2}\right|$.So $\left|z_{1}\right| \leq\left|z_{2}\right|$. In particular, we will write $\mathrm{z}_{1} \preccurlyeq \mathrm{z}_{2}$ if $\mathrm{z}_{1} \neq \mathrm{z}_{2}$ and one of (i), (ii) and(iii) is satisfied. In this case $\left|z_{1}\right|<\left|z_{2}\right|$. We will write $\mathrm{z}_{1}<\mathrm{z}_{2}$ if and only if (iii) is satisfied.

Take into account some fundamental properties of the partial order $\preccurlyeq$ on $\mathbf{C}$ as follows.

(i) $0 \leqslant z_{1} \preccurlyeq z_{2}$, then $\left|z_{1}\right|<\left|z_{2}\right|$.

(ii) If $\mathrm{z}_{1} \leqslant \mathrm{z}_{2}, \mathrm{z}_{2}<\mathrm{z}_{3}$, then $\mathrm{z}_{1}<\mathrm{z}_{3}$.

(iii) If $z_{1} \leqslant z_{2}$ and $\lambda \geq 0$ is a real number, then $\lambda z_{1} \leqslant \lambda_{2}$.

Definition1.[3] The "max" function for the partial order relation "ミ" is defined by the following.

(i) $\max \left\{\mathrm{z}_{1}, \mathrm{z}_{2}\right\}=\mathrm{z}_{2}$ if and only if $\mathrm{z}_{1} \leqslant \mathrm{z}_{2}$.

(ii) If $\mathrm{z}_{1} \leqslant \max \left\{\mathrm{z}_{2}, \mathrm{z}_{3}\right\}$, then $\mathrm{z}_{1} \leqslant \mathrm{z}_{2}$ or $\mathrm{z}_{1} \preccurlyeq \mathrm{z}_{3}$.

(iii) $\max \left\{\mathrm{z}_{1}, \mathrm{z}_{2}\right\}=\mathrm{z}_{2}$ if and only if $\mathrm{z}_{1} \leqslant \mathrm{z}_{2}$ or $\left|\mathrm{z}_{1}\right| \leq\left|\mathrm{z}_{2}\right|$.

Using Definition1 one can have the following lemma.

Lemma2 [3] Let $\mathrm{z}_{1}, \mathrm{z}_{2}, \mathrm{z}_{3} \ldots \in \mathbf{\in}$ and the partial order relation $\preccurlyeq$ is defined on $\mathbf{C}$. Then the following conditions are easy follow.

(i) If $\mathrm{z}_{1} \preccurlyeq \max \left\{\mathrm{z}_{2}, \mathrm{z}_{3}\right\}$, then $\mathrm{z}_{1} \preccurlyeq \mathrm{z}_{2}$ if $\mathrm{z}_{3} \leqslant \mathrm{z}_{2}$.

(ii) If $\mathrm{z}_{1} \leqslant \max \left\{\mathrm{z}_{2}, \mathrm{z}_{3}, \mathrm{z}_{4}\right\}$, then $\mathrm{z}_{1} \leq \mathrm{z}_{2}$ if $\max \left\{\mathrm{z}_{3}, \mathrm{z}_{4}\right\} \preccurlyeq \mathrm{z}_{2}$.

(iii) If $\mathrm{z}_{1} \leqslant \max \left\{\mathrm{z}_{2}, \mathrm{z}_{3}, \mathrm{z}_{4}, \mathrm{z}_{5}\right\}$, then $\mathrm{z}_{1} \leq \mathrm{z}_{2}$ if $\max \left\{\mathrm{z}_{3}, \mathrm{z}_{4}, \mathrm{z}_{5}\right\} \preccurlyeq \mathrm{z}_{2}$. 
Now we give the definition of complex valued metric space which has been introduced by Azam et. al. [1]

Definition3.Let $X$ be non empty set. If a mapping $\mathrm{d}: \mathrm{X} \times \mathrm{X} \rightarrow \mathbf{C}$ satisfies

(i) $0 \preccurlyeq d(x, y)$ for all $x, y \in X$ and $d(x, y)=0$ if and only if $x=y$,

(ii) $d(x, y)=d(y, x)$ for all $x, y \in X$,

(iii) $\mathrm{d}(\mathrm{x}, \mathrm{y}) \preccurlyeq \mathrm{d}(\mathrm{x}, \mathrm{z})+\mathrm{d}(\mathrm{z}, \mathrm{y})$ for all $\mathrm{x}, \mathrm{y}, \mathrm{z} \in \mathrm{X}$.

Then $d$ is called a complex valued metric on $X$ and the $\operatorname{pair}(X, d)$ is called complex valued metric space .

Let $\left\{x_{n}\right\}$ be a sequence in complex valued metric space $X$ and $x \in X$.If for every $\varepsilon \in \mathbf{C w i t h} 0<\varepsilon$ there $N \in \mathbf{N}$ such that, for all $\mathrm{n}>\mathrm{N}, \mathrm{d}\left(\mathrm{x}_{\mathrm{n}}, \mathrm{x}\right)<\varepsilon$, then $\mathrm{x}$ is called the limit of $\left\{\mathrm{x}_{\mathrm{n}}\right\}$ and is written as $\lim _{n \rightarrow \infty} \mathrm{x}_{\mathrm{n}}=\mathrm{x}$ as $n \rightarrow \infty$.If for every $\varepsilon \in \mathbf{C}$ with $0<\varepsilon$ there $N \in \mathbf{N}$ such that, for all $n>N, d\left(x_{n}, x_{m}\right)<\varepsilon$, then $\left\{x_{n}\right\}$ is called a Cauchy sequence in $X$. If every Cauchy sequence is convergent in $\mathrm{X}$, then $\mathrm{X}$ is called a complete complex valued metric space.

Lemma2. [1] Let $(X, d)$ is called complex valued metric space and let $\left\{x_{n}\right\}$ be a sequence in $X$. Then

(i) $\left\{x_{n}\right\}$ converges to $x$ if and only if $\left|d\left(x_{n}, x\right)\right| \rightarrow 0$ as $n \rightarrow \infty$.

(ii) $\left\{x_{n}\right\}$ is Cauchy sequence if and only if $\left|d\left(x_{n}, x_{m}\right)\right| \rightarrow 0$ as $\rightarrow \infty$.

\section{Main Results}

In this paper, we prove Generalized contraction principle in complex valued metric space as follows:

Theorem1.1. Let $\mathrm{T}: \mathrm{X} \rightarrow \mathrm{X}$ be self mappings of a complex valued metric space(X,d) satisfying

$\mathrm{d}(\mathrm{Tx}, \mathrm{Ty}) \preccurlyeq \mathrm{k} \mathrm{M}(\mathrm{x}, \mathrm{y}) \quad$ where $\mathrm{k} \in[0,1)$

where $\mathrm{M}(\mathrm{x}, \mathrm{y})=\max \left\{\mathrm{d}(\mathrm{x}, \mathrm{y}), \mathrm{d}(\mathrm{Tx}, \mathrm{y}), \mathrm{d}(\mathrm{Ty}, \mathrm{y}), \frac{[d(T x, y)+d(T y, x)]}{2}\right\}{ }^{[5]}$

Then $\mathrm{T}$ has a unique fixed point.

Proof. Let $x_{0} \in X$ be arbitrary point and define a sequence $\left\{x_{n}\right\}$ as $T x_{n}=x_{n+1}$. Then putting $x=x_{n}, y=x_{n-1}$ we get $\mathrm{d}\left(\mathrm{x}_{\mathrm{n}+1}, \mathrm{x}_{\mathrm{n}}\right)=\mathrm{d}\left(\mathrm{Tx}_{\mathrm{n}}, \mathrm{Tx}_{\mathrm{n}-1}\right) \preccurlyeq \mathrm{k} \mathrm{M}\left(\mathrm{x}_{\mathrm{n}}, \mathrm{x}_{\mathrm{n}-1}\right)$

where $M\left(x_{n}, x_{n-1}\right)=\max \left\{d\left(x_{n}, x_{n-1}\right), d\left(T x_{n}, x_{n}\right), d\left(T x_{n-1}, x_{n-1}\right), \frac{\left[d\left(\operatorname{Tx}_{n}, x_{n}\right)+d\left(T x_{n-1}, x_{n}\right)\right]}{2}\right\}$.

$\mathrm{M}\left(\mathrm{x}_{\mathrm{n}}, \mathrm{x}_{\mathrm{n}-1}\right)=\max \left\{\mathrm{d}\left(\mathrm{x}_{\mathrm{n}}, \mathrm{x}_{\mathrm{n}-1}\right), \mathrm{d}\left(\mathrm{x}_{\mathrm{n}+1}, \mathrm{x}_{\mathrm{n}}\right), \mathrm{d}\left(\mathrm{x}_{\mathrm{n},}, \mathrm{x}_{\mathrm{n}-1}\right), \frac{\left[d\left(\mathrm{x}_{\mathrm{n}+1}, \mathrm{x}_{\mathrm{n}}\right)+d\left(x_{n}, x_{n}\right)\right]}{2}\right\}$.

$\preccurlyeq \max \left\{\mathrm{d}\left(\mathrm{x}_{\mathrm{n}}, \mathrm{x}_{\mathrm{n}-1}\right), \mathrm{d}\left(\mathrm{x}_{\mathrm{n}+1}, \mathrm{x}_{\mathrm{n}}\right)\right\}$.

Now from (2) we get

$\mathrm{d}\left(\mathrm{x}_{\mathrm{n}+1}, \mathrm{x}_{\mathrm{n}}\right)=\mathrm{d}\left(\mathrm{Tx}_{\mathrm{n}}, \mathrm{Tx}_{\mathrm{n}-1}\right) \preccurlyeq \mathrm{k} \max \left\{\mathrm{d}\left(\mathrm{x}_{\mathrm{n}}, \mathrm{x}_{\mathrm{n}-1}\right), \mathrm{d}\left(\mathrm{x}_{\mathrm{n}+1}, \mathrm{x}_{\mathrm{n}}\right)\right\}$.

$\left|\mathrm{d}\left(\mathrm{x}_{\mathrm{n}+1}, \mathrm{x}_{\mathrm{n}}\right)\right| \leq \mathrm{k}\left|\max \left\{\mathrm{d}\left(\mathrm{x}_{\mathrm{n}}, \mathrm{x}_{\mathrm{n}-1}\right), \mathrm{d}\left(\mathrm{x}_{\mathrm{n}+1}, \mathrm{x}_{\mathrm{n}}\right)\right\}\right|$

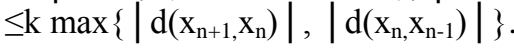

We shall take two cases.

Suppose $\left|d\left(x_{n+1}, x_{n}\right)\right|>\left|d\left(x_{n}, x_{n-1}\right)\right|$. Since $\left|d\left(x_{n+1}, x_{n}\right)\right|>0$, we obtain

$\left|\mathrm{d}\left(\mathrm{x}_{\mathrm{n}+1}, \mathrm{x}_{\mathrm{n}}\right)\right| \leq \mathrm{k}\left|\mathrm{d}\left(\mathrm{x}_{\mathrm{n}+1}, \mathrm{x}_{\mathrm{n}}\right)\right|$ a contradiction. Therefore, we get

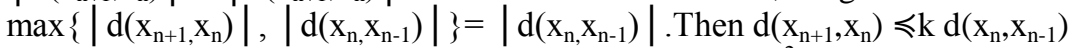

Again $d\left(x_{n}, x_{n-1}\right) \preccurlyeq k d\left(x_{n-1}, x_{n-2}\right)$, then from $(3) d\left(x_{n+1}, x_{n}\right) \preccurlyeq k^{2} d\left(x_{n-1}, x_{n-2}\right)$.

Continuing in the same manner, we have $\mathrm{d}\left(\mathrm{x}_{\mathrm{n}+1}, \mathrm{x}_{\mathrm{n}}\right) \preccurlyeq \mathrm{k}^{\mathrm{n}} \mathrm{d}\left(\mathrm{x}_{1}, \mathrm{x}_{0}\right)$.

Then for all $n, m \in N$ and repeated use of triangular inequality for $m \geq 1$ and from (5), we have

$$
\begin{aligned}
& \mathrm{d}\left(\mathrm{x}_{\mathrm{n}+\mathrm{m}}, \mathrm{x}_{\mathrm{n}}\right) \preccurlyeq \mathrm{d}\left(\mathrm{x}_{\mathrm{n}+\mathrm{m}}, \mathrm{x}_{\mathrm{n}+\mathrm{m}-1}\right)+\ldots \ldots \ldots \ldots . .+\mathrm{d}\left(\mathrm{x}_{\mathrm{n}+1}, \mathrm{x}_{\mathrm{n}}\right) \\
& \preccurlyeq \sum_{p=n}^{n+m-1} k^{n} d\left(x_{1}, x_{0}\right) \\
& \preccurlyeq \sum_{p=n}^{\infty} k^{n} d\left(x_{1}, x_{0}\right)
\end{aligned}
$$

Since $k \in[0,1)$, if we take limit as $n \rightarrow \infty$ then $\left|d\left(x_{n+m}, x_{n}\right)\right| \rightarrow 0$.

So, $\left\{x_{n}\right\}$ is complex valued Cauchy sequence .By completeness of $(X, d)$ there exists $z \in X$ such that $\left\{x_{n}\right\}$ is complex valued convergent to $\mathrm{z}$.

Next we prove $T z=z$. Assume on contrary that $T z \neq z$. Then by (1), put $x=z, y=x_{n+1}$

$\mathrm{d}\left(\mathrm{Tz}, \mathrm{Tx}_{\mathrm{n}+1}\right) \preccurlyeq \mathrm{k} \mathrm{M}\left(\mathrm{z}, \mathrm{x}_{\mathrm{n}+1}\right)$

where $\mathrm{M}\left(\mathrm{z}, \mathrm{x}_{\mathrm{n}+1}\right)=\max \left\{\mathrm{d}\left(\mathrm{z}, \mathrm{x}_{\mathrm{n}+1}\right), \mathrm{d}(\mathrm{Tz}, \mathrm{z}), \mathrm{d}\left(\mathrm{Tx}_{\mathrm{n}+1}, \mathrm{x}_{\mathrm{n}+1}\right), \frac{\left[d\left(\mathrm{Tz}, \mathrm{x}_{\mathrm{n}+1}\right)+d\left(x_{n}, \mathrm{z}\right)\right]}{2}\right\}$.

As $\left\{x_{n}\right\}$ is convergent to $z$, therefore, $\lim _{n \rightarrow \infty}|d(z, x)|=\lim _{n \rightarrow \infty}|d(x, x)|=0$.

Thus letting $\mathrm{n} \rightarrow \infty, \mathrm{d}(\mathrm{Tz}, \mathrm{z}) \leqslant \mathrm{kd}(\mathrm{Tz}, \mathrm{z})$ that is $|\mathrm{d}(\mathrm{Tz}, \mathrm{z})| \leq|\mathrm{d}(\mathrm{Tz}, \mathrm{z})|$ which is contradiction.

$\mathrm{So}, \mathrm{Tz}=\mathrm{z}$ that is, $\mathrm{z}$ is fixed point of $\mathrm{T}$.

Uniqueness. Let $u(u \neq z)$ be another fixed point of $T$, then from (2) we have $\mathrm{d}(\mathrm{u}, \mathrm{z})=\mathrm{d}(\mathrm{Tu}, \mathrm{Tz}) \preccurlyeq \mathrm{k} \mathrm{M}(\mathrm{u}, \mathrm{z})$

where $\mathrm{M}(\mathrm{u}, \mathrm{z})=\max \left\{\mathrm{d}(\mathrm{u}, \mathrm{z}), \mathrm{d}(\mathrm{Tu}, \mathrm{u}), \mathrm{d}(\mathrm{Tz}, \mathrm{z}), \frac{[d(\mathrm{Tu}, \mathrm{z})+d(T z, u)]}{2}\right\}$. 
$=\mathrm{d}(\mathrm{u}, \mathrm{z})$

$|\mathrm{d}(\mathrm{u}, \mathrm{z})| \leq \mathrm{k}|\mathrm{d}(\mathrm{u}, \mathrm{z})|$ which is a contradiction. Hence $\mathrm{u}=\mathrm{z}$ that is, $\mathrm{T}$ has a unique fixed point.

\section{References}

[1]. Azam, A. Fisher, and Khan, M. Common fixed point theorems in complex valued metric space, Numerical Functional Analysis and Optimization,vol.32,pp.243-253,2011.

[2]. Banach, S. Sur les operations dans les ensembles etleur application aux equations integrals ,Fundamenta Mathematicae,vol.3,pp.133-181,1992.

[3]. Rouzkard, F. And Imdad, M. Some common fixed point theorems on complex valued metric space, Computers \& Mathematics with Applications,vol.64, no.6,pp.1866-1874,2012.

[4]. Verma ,R.K. and Pathak, H.K. Some common fixed point theorems using property(E.A) in complex valued metric space, Thai journal of Mathematics,2012.

[5]. Karapinar, E, Samet, B: Generalized $\alpha-\psi$ contractive type mappings and related fixed point theorems with applications. Abstr. Appl. Anal.2012, Article ID 793486 (2012). doi:10.1155/2012/793486. 